\title{
TRACKING THE POST-BBN EVOLUTION OF DEUTERIUM
}

\author{
Gary Steigman \\ Departments of Physics and Astronomy \\ Center for Cosmology and Astro-Particle Physics \\ The Ohio State University \\ 191 West Woodruff Avenue \\ Columbus, OH 43210, USA
}

\begin{abstract}
The primordial abundance of deuterium produced during Big Bang Nucleosynthesis (BBN) depends sensitively on the universal ratio of baryons to photons, an important cosmological parameter probed independently by the Cosmic Microwave Background (CMB) radiation. Observations of deuterium in high-redshift, low-metallicity QSO Absorption Line Systems (QSOALS) provide a key baryometer, determining the baryon abundance at the time of BBN to a precision of $\sim 5 \%$. Alternatively, if the CMB-determined baryon to photon ratio is used in the BBN calculation of the primordial abundances, the BBN-predicted deuterium abundance may be compared with the primordial value inferred from the QSOALS, testing the standard cosmological model. In the post-BBN universe, as gas is cycled through stars, deuterium is only destroyed so that its abundance measured anytime, anywhere in the Universe, bounds the primordial abundance from below. Constraints on models of post-BBN Galactic chemical evolution follow from a comparison of the relic deuterium abundance with the FUSE-inferred deuterium abundances in the chemically enriched, stellar processed material of the local ISM.
\end{abstract}

Keywords: Deuterium, Galactic Chemical Evolution, Big Bang Nucleosynthesis

PACS: 98.35.Bd, 98.35.Mp, 98.58.Ay, 26.35.+c, 98.80.Ft

\section{INTRODUCTION}

Of the light nuclides synthesized in astrophysically interesting abundances $\left(\mathrm{D},{ }^{3} \mathrm{He}\right.$, ${ }^{4} \mathrm{He},{ }^{7} \mathrm{Li}$ ) during Big Bang Nucleosynthesis (BBN), the post-BBN evolution of deuterium is the simplest. As gas is cycled through stars, deuterium is only destroyed [1], so that its abundance, observed anywhere in the Universe, at any time in its evolution, is constrained to be bounded from above by its primordial, BBN abundance: $(\mathrm{D} / \mathrm{H})_{\mathrm{OBS}} \leq(\mathrm{D} / \mathrm{H})_{\mathrm{P}}$. In systems at high redshift and/or with very low metallicity, the observed deuterium abundance should approach its primordial value. Its simple post$\mathrm{BBN}$ evolution, along with the sensitivity of its predicted $\mathrm{BBN}$ abundance to the baryon to photon ratio $\eta_{\mathrm{B}} \equiv n_{\mathrm{B}} / n_{\gamma}\left((\mathrm{D} / \mathrm{H})_{\mathrm{P}} \propto \eta_{\mathrm{B}}^{-1.6}\right)$, identifies deuterium as the baryometer of choice. The baryon mass density parameter, $\Omega_{\mathrm{B}}$ (the fraction of the present critical mass density contributed by baryons), is related to $\eta_{\mathrm{B}}$ and the present value of the Hubble parameter, $H_{0} \equiv 100 h \mathrm{kms}^{-1} \mathrm{Mpc}^{-1}$, by $\Omega_{\mathrm{B}} h^{2}=\eta_{10} / 274$, where $\eta_{10} \equiv 10^{10} \eta_{\mathrm{B}}$. Given the dependence of $(\mathrm{D} / \mathrm{H})_{\mathrm{P}}$ on $\eta_{\mathrm{B}}$, observations which constrain the relic deuterium abundance to, say, $\sim 10 \%$, lead to a $\sim 6 \%$ measurement of the baryon to photon ratio when the Universe was only a few minutes old. Alternatively, the baryon to photon ratio determined by observations of the cosmic microwave background (CMB), which probe a 


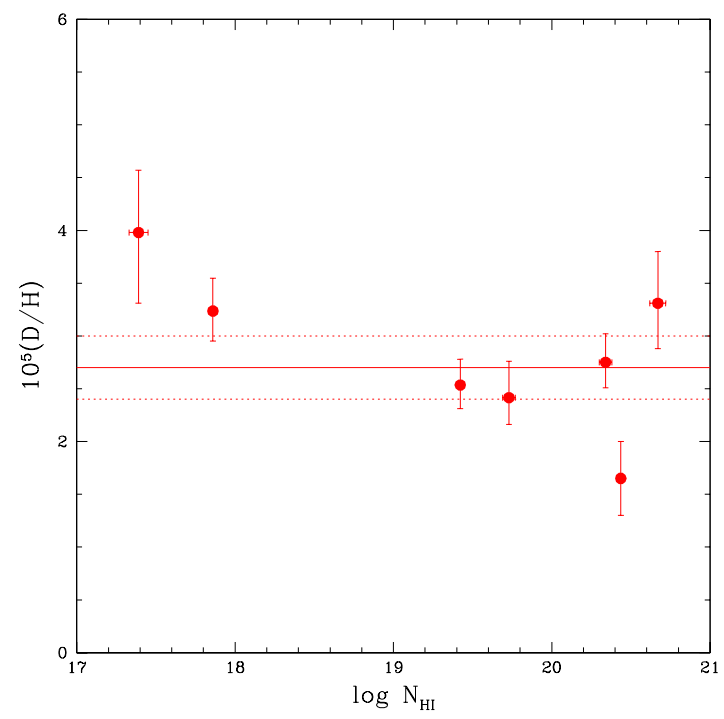

FIGURE 1. Deuterium abundances, the $\mathrm{D}$ to $\mathrm{H}$ ratios by number, derived from observations of high redshift, low metallicity QSO Absorption Line Systems, are shown as a function of the corresponding neutral hydrogen, $\mathrm{H}$ I, column densities. The solid line shows the weighted mean of the $\mathrm{D} / \mathrm{H}$ ratios and the dashed lines indicate the $\pm 1 \sigma$ error estimates; see the text.

time when the Universe was some 400 thousand years old, may be used in the BBN calculation to predict the primordial D abundance, which may then be compared to the relic value inferred from observations of deuterium at high redshifts in systems of very low metallicity, testing the standard model of cosmology.

In the post-BBN Universe, the $\mathrm{D}$ "astration" factor, $f_{\mathrm{D}} \equiv(\mathrm{D} / \mathrm{H})_{\mathrm{P}} /(\mathrm{D} / \mathrm{H})$, measures the virgin fraction $\left(1 / f_{\mathrm{D}}\right) ; 1 / f_{\mathrm{D}}$ is the fraction of gas which has never been processed through stars. Due to stellar nucleosynthesis, as the metallicity, $Z$, in a system increases, the deuterium abundance should decrease, suggesting that, in the absence of dust depletion, the observed values of $\mathrm{D} / \mathrm{H}$ and $Z$ should be anti-correlated.

\section{BBN AND THE PRIMORDIAL ABUNDANCE OF DEUTERIUM}

In Figure 1 are shown the deuterium abundances inferred from observations of seven, high redshift, low metallicity QSO Absorption Line Systems (QSOALS) [2]. The weighted mean of these abundance determinations provides an estimate of the primordial deuterium abundance, $y_{\mathrm{DP}} \equiv 10^{5}(\mathrm{D} / \mathrm{H})_{\mathrm{P}}=2.7 \pm 0.2$. Since the dispersion of the individual abundance determinations around the mean value is very large, the formal error in the mean has been multiplied by the square root of the reduced $\chi^{2}$ to provide a more realistic error estimate.

From $\mathrm{BBN}$ [3], for $y_{\mathrm{DP}}=2.7 \pm 0.2$, the baryon abundance is determined, at the $\sim 5 \%$ level, to be $\eta_{10}=6.0 \pm 0.3$. This determination, some $\sim 20$ minutes after the expansion has begun, is in excellent agreement with the value determined independently from the $\mathrm{CMB}$, some $400 \mathrm{kyr}$ later, $\eta_{10}=6.1 \pm 0.2$ (see Simha \& Steigman [3] and references 


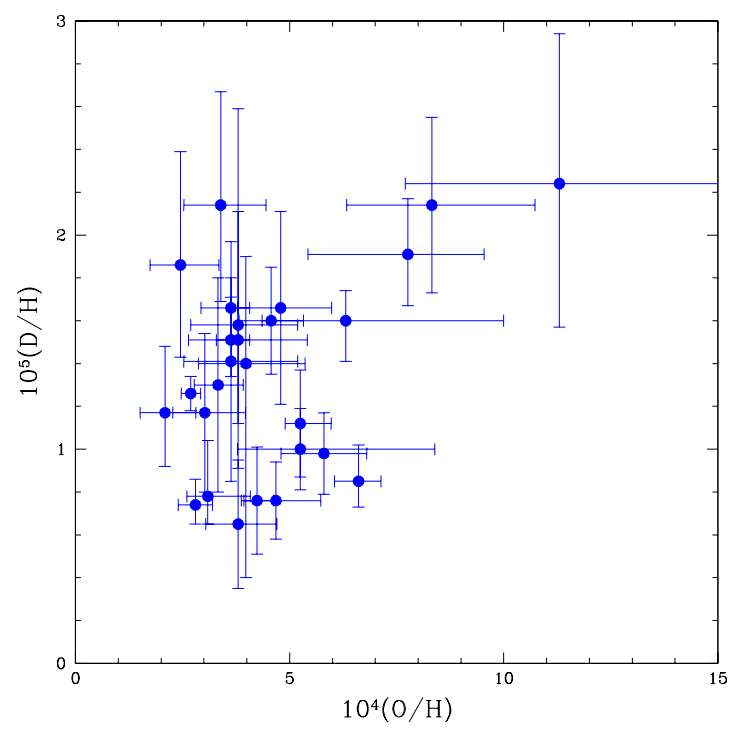

FIGURE 2. The FUSE-observed ISM abundances of deuterium versus those of oxygen observed along the same lines of sight; from [7].

therein). If the CMB-determined value of the baryon abundance is used to predict the primordial abundance of deuterium, the result is $y_{\mathrm{DP}}=2.6 \pm 0.1$, in perfect agreement, within the estimated errors, with the relic abundance inferred from the QSOALS data.

\section{OBSERVATIONS OF D IN THE ISM}

The conventional wisdom (ante?) has been that mixing of the ISM within $1-2 \mathrm{kpc}$ of the location of the solar system is efficient so that this relatively local gas is wellmixed with homogenized abundances. In particular, the conventional wisdom led to the expectation that observations of deuterium and, e.g., oxygen within the local $(\sim 1 \mathrm{kpc})$ ISM would reveal uniform abundances, "the interstellar abundances", of these (and other) elements. Beginning with the pioneering Copernicus observations [4], continuing with observations provided by the IUE satellite, and the GHRS and STIS instruments onboard the HST, and confirmed by those of the FUSE mission [5], the data reveal that nothing could be further from the truth. As may be seen in Figure 2, the deuterium abundances inferred from observations in the relatively local ISM (see [5] for details and further references) vary over a range of a factor of $\sim 3$, while those of oxygen range over a factor of $\sim 4$.

While there has not been much discussion in the literature of the factor $\sim 4$ range in oxygen abundances observed along lines of sight (LOS) within $\sim 1-2 \mathrm{kpc}$ of the Sun, much attention has been paid to the factor of $\sim 3$ range in the deuterium abundances. The general consensus is that the large range in observationally-inferred deuterium abundances is the result of preferential depletion onto grains of D, relative to $H$ [6]. Although preferential depletion of $\mathrm{D}$ is likely the correct explanation for most, possibly 


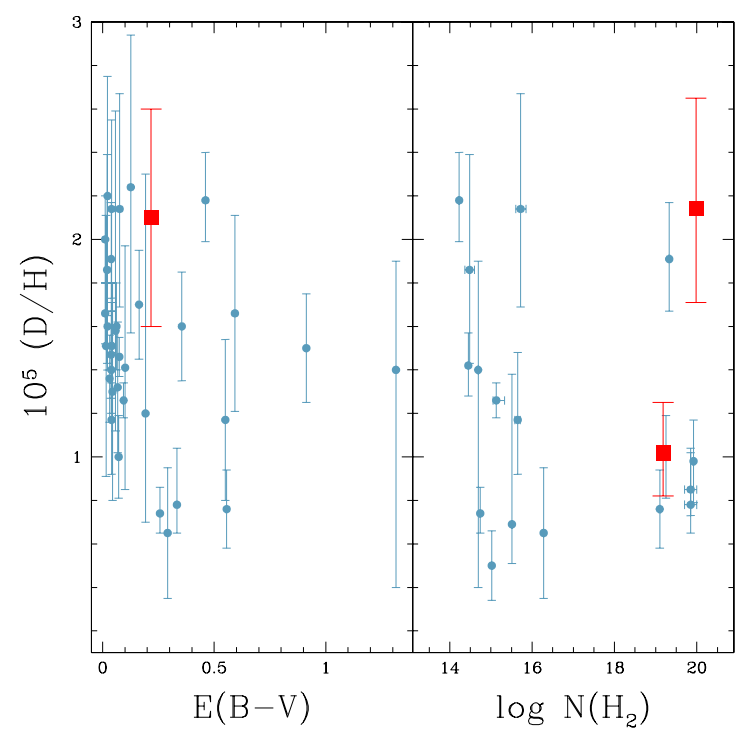

FIGURE 3. In the left-hand panel the FUSE observed D/H ratios are shown versus the corresponding LOS values of $\mathrm{E}(\mathrm{B}-\mathrm{V})$ and, in the right-hand panel of $\log \mathrm{N}\left(\mathrm{H}_{2}\right)$; from [7].

all, of the observed variation in ISM D abundances, there is, in fact, surprisingly little independent data in support of it. For example, if depletion onto grains is the culprit, it might be expected that the $\mathrm{D} / \mathrm{H}$ ratios would correlate (or anticorrelate) with the reddening or, with the amount of molecular hydrogen. However, as the two panels of Figure 3 reveal, both high and low D abundances are found at very small values of $\mathrm{E}(\mathrm{B}-\mathrm{V})$ and of $\mathrm{N}\left(\mathrm{H}_{2}\right)$ and, while there may be weak evidence in favor of decreasing $\mathrm{D}$ abundances at high $\mathrm{E}(\mathrm{B}-\mathrm{V})$, both high and low $\mathrm{D} / \mathrm{H}$ values are found along the LOS with higher $\mathrm{N}\left(\mathrm{H}_{2}\right)$. This absence of a smoking gun for depletion is also revealed in Figure 4 where the D abundances are shown as a function of the corresponding iron abundances along the same LOS. While there IS evidence of a correlation between D/H and $\mathrm{Fe} / \mathrm{H}$ at low $\mathrm{Fe}$ abundances, that trend disappears for $\log y_{\mathrm{Fe}} \equiv 10^{6}(\mathrm{Fe} / \mathrm{H}) \gtrsim 0$, hinting that along those LOS with $\log y_{\mathrm{Fe}} \gtrsim 0$, deuterium may be undepleted.

Whatever is the correct explanation for the large range in the ISM abundances of D and $\mathrm{O}$, it is clear that the local ISM is NOT homogeneous. Since any local depletion of D onto grains has not been homogenized in the ISM, it is not unreasonable to suspect that there might also be some LOS along which infalling, D-enhanced, O-poor gas has been incompletely mixed with the processed, D-astrated, O-enhanced gas already present [7]. This latter effect is illustrated in Figure 4 by the solid curve which shows the effect of unmixed, nearly primordial infall [7] to yield higher D and lower Fe abundances than is predicted by the fiducial Galactic chemical evolution (GCE) model, shown by the star. Abundances to the left and below the dashed lines may have resulted from $\mathrm{Fe}$ and $\mathrm{D}$ depletion respectively, while any $\mathrm{D}$ abundances above the dashed line may have been contaminated by infall. These two competing processes, depletion onto grains and infall, complicate the attempt to use the data to infer the "true" ISM abundances of D and Fe (and $\mathrm{O}$ ). 


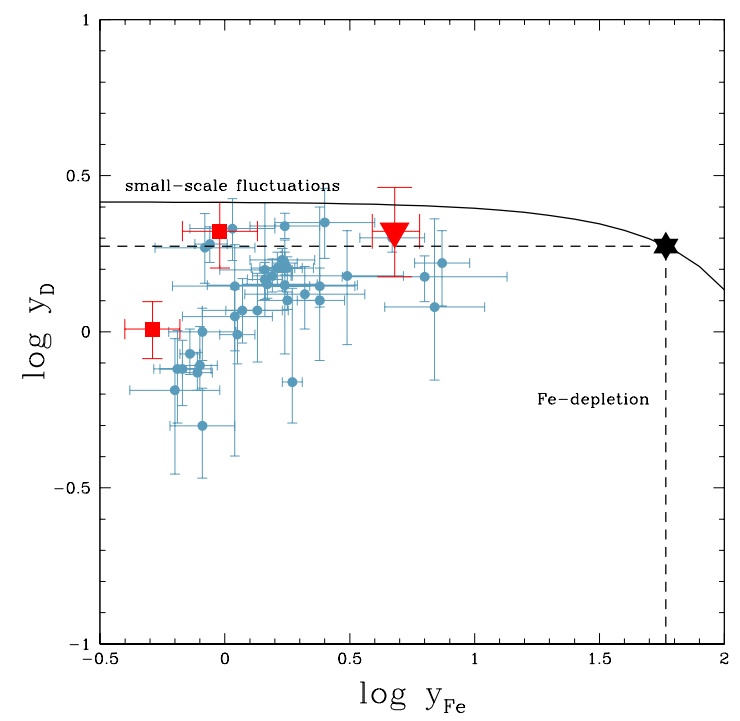

FIGURE 4. FUSE observed ISM D and Fe abundances $\left(y_{\mathrm{D}} \equiv 10^{5}(\mathrm{D} / \mathrm{H}), y_{\mathrm{Fe}} \equiv 10^{6}(\mathrm{Fe} / \mathrm{H})\right)$; from [7]. The star shows the ISM abundances predicted by the fiducial GCE model (see the text). D and Fe abundances below and to the left of the dashed lines are consistent with depletion. The solid curve shows the effect of local abundance fluctuations resulting from incompletely mixed infall of D-enhanced, Fe-free material.

\section{GALACTIC CHEMICAL EVOLUTION}

Unable to survive the high temperatures in stars, deuterium is destroyed when gas is incorporated in stars. As gas cycles through stars and the stellar processed material is returned to the interstellar medium (ISM) of the Galaxy, the metallicity of the ISM increases while the abundance of deuterium decreases. Infall to the disk of the galaxy of essentially unprocessed gas plays a crucial role in the chemical evolution of the Galaxy [8]. Infall of primordial (or very nearly primordial) gas which is metal-free (or very nearly so) and whose deuterium abundance is primordial (or very nearly so) dilutes the metallicity of the ISM while enhancing its D abundance [7, 9, 10].

In Steigman, Romano, Tosi (SRT) [7], we considered GCE models with several different prescriptions for the stellar IMF and stellar lifetimes and predicted the ISM D astration factor and the current ISM oxygen (and iron) abundances. For those GCE models consistent with other independent, observational data, SRT found that $1.4 \lesssim f_{\mathrm{D}} \lesssim 1.8$ and that $7 \lesssim y_{\mathrm{O}} \lesssim 12$, where $y_{\mathrm{O}} \equiv 10^{4}(\mathrm{O} / \mathrm{H})$. For our fiducial model, SRT adopted the Scalo IMF [11] along with the Schaller et al. [12] prescription for stellar lifetimes, corresponding to $f_{\mathrm{D}}=1.39$ and $y_{\mathrm{O}}=8.4$; these values are indicated by the stars in Figures 4 and 5. As noted by SRT if recent infall of unprocessed material were not fully mixed in the ISM along some LOS, there would be an anticorrelation between the $\mathrm{D}$ and $\mathrm{O}$ abundances along such contaminated LOS, predicting $y_{\mathrm{D}}^{\mathrm{OBS}}=2.61-0.087 y_{\mathrm{O}}^{\mathrm{OBS}}$ (the dashed line in the upper panel of Fig. 5). SRT also noted that since some of the dispersion observed among the $\mathrm{D}$ and $\mathrm{O}$ abundances may be due to systematic errors in the $\mathrm{H}$ I column densities, the D/O ratio would be unaffected by such errors. For LOS contaminated by 


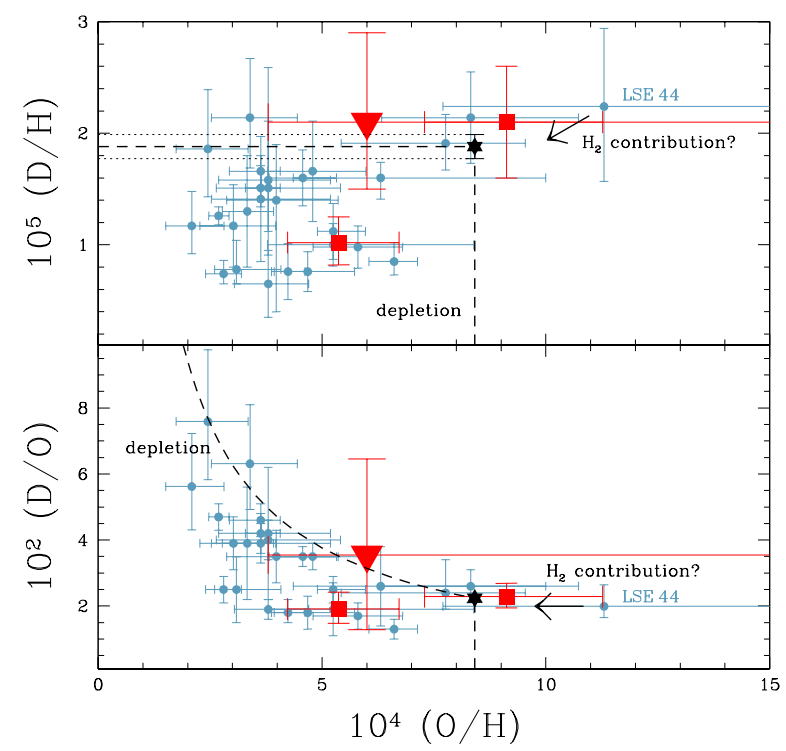

FIGURE 5. The upper panel shows the FUSE observed deuterium and oxygen abundances, while the lower panel plots the $\mathrm{D}$ to $\mathrm{O}$ ratios as functions of the oxygen abundances; from [7]. The stars show the ISM D and D/O versus $O$ abundances predicted by the fiducial GCE model (see the text). Abundances below and to the left of the dashed lines are consistent with $\mathrm{D}$ and $\mathrm{O}$ depletion.

unmixed infall, $10(\mathrm{D} / \mathrm{O}) \equiv y_{\mathrm{D}} / y_{\mathrm{O}}, 10(\mathrm{D} / \mathrm{O})=26.1-0.87$ (the dashed line in the lower panel of Fig. (5).

\section{TOWARDS AN ESTIMATE OF THE D ASTRATION FACTOR}

Estimating the Galactic D astration factor is complicated by the possibilities that the "true" ISM D abundance along some LOS may have been reduced by preferential D depletion onto grains and/or, enhanced by incompletely mixed infall of gas with the higher, primordial D abundance. Linsky et al. [5] assume that unmixed infall makes no contribution at all so that, allowing for depletion, the maximum of the ISM D/H ratios provides a lower limit to the "true" ISM D abundance. Linsky et al. then find the weighted mean of $\mathrm{D} / \mathrm{H}$ for the 5 highest $\mathrm{D}$ abundances, concluding that $y_{\mathrm{D}}^{\mathrm{ISM}} \geq$ $y_{\mathrm{D}}^{\mathrm{MAX}}=2.17 \pm 0.017$ (or, including a correction for the Local Bubble D abundance, $\left.y_{\mathrm{D}}^{\mathrm{MAX}}=2.37 \pm 0.024\right)$. These choices correspond to astration factors $f_{\mathrm{D}} \lesssim 1.1-1.2$, considerably smaller than those predicted by the GCE models, $f_{\mathrm{D}} \approx 1.4-1.8$ [7]. In contrast, SRT allow for the possibility that the highest observed $\mathrm{D} / \mathrm{H}$ ratios may represent the "true" ISM D abundance, although unmixed infall may have contaminated the D abundance along some LOS and, they note that there are many more than 5 LOS with high $\mathrm{D} / \mathrm{H}$ abundances which are equal within the errors. SRT find that for the 18 FUSE LOS with the highest $\mathrm{D} / \mathrm{H}$ ratios $\left(y_{\mathrm{D}} \geq 1.5\right)$, nine have $y_{\mathrm{D}} \geq 1.9$ and nine have $y_{\mathrm{D}} \leq 1.7$, and they find for the weighted mean, $y_{\mathrm{D}}^{\mathrm{ISM}}=1.9$. For the primordial abundance $y_{\mathrm{DP}}=2.7 \pm 0.2$, this corresponds to $f_{\mathrm{D}}=1.4 \pm 0.1$, in agreement with the fiducial model. 


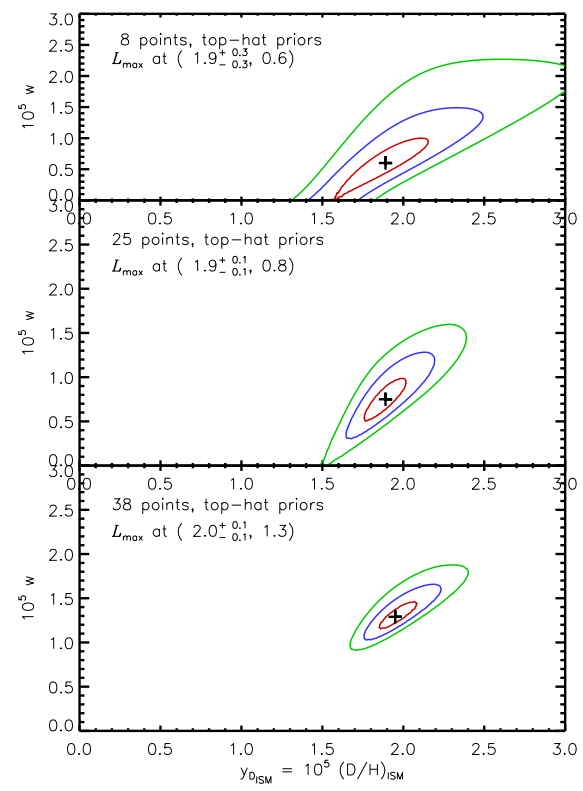

FIGURE 6. The $68 \%, 95 \%$ and $99 \%$ contours in the $y_{\mathrm{D}}-w$ plane $\left(y_{\mathrm{D}} \equiv 10^{5}(\mathrm{D} / \mathrm{H})_{\mathrm{ISM}}\right)$ for a top-hat prior distribution for three subsets of the FUSE D/H data; see the text for details. From [13].

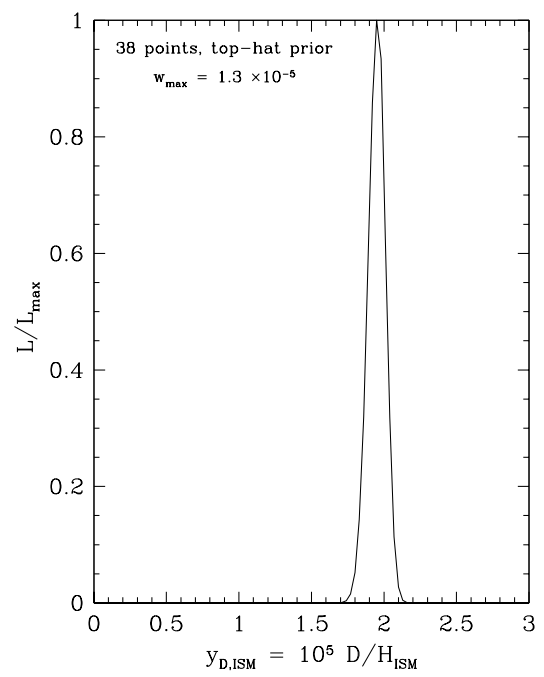

FIGURE 7. The likelihood distribution for the ISM D abundance $\left(y_{\mathrm{D}, \mathrm{ISM}}=1.95_{-0.09}^{+0.15}\right)$ inferred from the data for 38 LOS (see Fig. 6), using the top-hat prior for $w$. From [13].

Recently, B. Fields, T. Prodanović, and I [13] have explored a different path to estimating $y_{\mathrm{D}}^{\mathrm{ISM}}$, using a Bayesian approach pioneered by Hogan, Olive, and Scully [14]. In our analysis we neglect unmixed infall and assume that the scatter among the FUSEobserved $\mathrm{D} / \mathrm{H}$ ratios is entirely due to preferential depletion of $\mathrm{D}$ onto grains, so that 
our estimate likely provides an upper bound to the true value of $y_{\mathrm{D}}^{\mathrm{ISM}}$. We assume that the gas phase D abundances (including uncertainties) are equal to the differences between the true ISM D abundance and corrections, $w \equiv y_{\mathrm{D}}^{\mathrm{ISM}}-y_{\mathrm{D}}^{\mathrm{OBS}}$, for depletion. We adopt a prior for the distribution of $w$ and maximize the likelihood to find the observed abundances as a function of $y_{\mathrm{D}}^{\mathrm{ISM}}$ and $w$. In Figure 6 are shown the contours in the $y_{\mathrm{D}}^{\text {ISM }}$ $-w$ plane for a top-hat distribution for $w$ for three subsets of the data. As the number of data points is increased, the contours shrink and $w>0$ is strongly favored, arguing in support of depletion. The likelihood distribution for $y_{\mathrm{D}}^{\mathrm{ISM}}=1.95_{-0.09}^{+0.15}$, derived from the top-hat prior for D/H along $38 \mathrm{LOS}$ [13], is shown in Figure 7] Combining this estimate for (a lower bound to) $y_{\mathrm{D}}^{\mathrm{ISM}}$ with the weighted mean of the QSOALS D/H ratios [2] from Simha \& Steigman [3], $y_{\mathrm{DP}}=2.70_{-0.20}^{+0.22}$, results in a $\mathrm{D}$ astration factor $f_{\mathrm{D}} \gtrsim 1.38_{-0.15}^{+0.13}$ which, within the uncertainties, is consistent with all models of GCE.

\section{ACKNOWLEDGMENTS}

I thank the organizers for the invitation to speak at this conference and the editors of these proceedings for their patience. Much of what I have presented here has resulted from collaborations with B. D. Fields, T. Prodanović, D. Romano, and M. Tosi, and I gladly acknowledge their help and advice; I hasten to add that any errors here are mine alone. My research is supported at The Ohio State University by a grant from the US Department of Energy. The work reported here was carried out when I was a Visiting Professor at IAG - USP in São Paulo, Brazil and was supported by a grant from FAPESP.

\section{REFERENCES}

1. R. J. Epstein, J. Lattimer, and D. N. Schramm, Nature, 263, 198 (1976); T. Prodanović and B. D. Fields, ApJ, 597, 48 (2003).

2. M. Pettini et al., MNRAS, 391, 1499 (2008).

3. J. P. Kneller and G. Steigman, New J. Phys., 6, 117 (2004); G. Steigman, Int. J. Mod. Phys., E15, 1 (2006); G. Steigman, Ann. Rev. Nucl. Part. Sci., 57, 463 (2007); V. Simha and G. Steigman, JCAP, 06, 016 (2008).

4. D. G. York and J. B. Rogerson ApJ, 203378 (1976); C. Laurent, A. Vidal-Madjar, and D. G. York, ApJ, 229, 923 (1979); R. Ferlet, A. Vidal-Madjar, C. Laurent, and D. G. York ApJ, 242, 576 (1980).

5. J. L. Linsky et al., ApJ, 647, 1106 (2006); C. M. Oliveira, H. W. Moos, P. Chayer, and J. W. Kruck, ApJ, 642, 283 (2006).

6. M. Jura, in Advances in UV Astronomy, p. 54 [Y. Kondo, J. Mead, and R. D. Chapman, eds., NASA, Washington] (1982); B. T. Draine, in Origin and Evolution of the Elements, p. 317 [A. McWilliam and M. Rauch, eds., Cambridge Univ. Press, Cambridge] (2004).

7. G. Steigman, D. Romano, and M. Tosi, MNRAS, 378, 576 (2007).

8. M. Tosi, $A \& A, 197,33$ (1988); ibid A\&A, 197, 47 (1988).

9. G. Steigman and M. Tosi, ApJ, 401, 150 (1992); ibid ApJ, 453, 173 (1995); E. Vangioni-Flam, K. A. Olive, and N. Prantzos, ApJ, 427, 618 (1994); N. Prantzos, A\&A, 310, 106 (1996); M. Tosi, G.

Steigman, F. Matteucci, and C. Chiappini, ApJ, 498, 226 (1998).

10. T. Prodanović and B. D. Fields, JCAP, 09, 003 (2008).

11. J. M. Scalo, Fund. Cosm. Phys., 11, 1 (1986).

12. G. Schaller, D. Schaerer, G. Meynet, and A. Maeder, A\&AS, 96, 269 (1992).

13. T. Prodanović, B. D. Fields, and G. Steigman, In preparation (2008).

14. C. J. Hogan, K. A. Olive, and S .T. Scully, ApJL, 489, L119 (1997). 